\title{
JOURNAL.RU
}

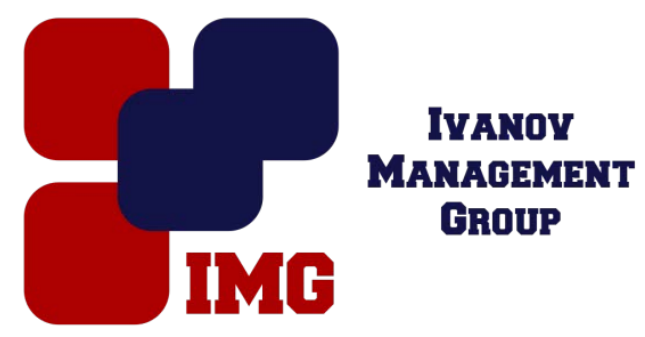

Шардаков В.М., Болодурина И.П.

Оренбургский государственный университет Оренбург, Россия

doi: 10.18411/lj-30-06-2017-17

idsp 000001:lj-30-06-2017-17

\section{Основные принципы генерации ландшафтных карт с применением графической библиотеки}

\section{Введение}

Для реализации ландшафтной генерации, проведем сравнительный анализ графических библиотек DirectX и OpenGL, которые могут быть использованы для решения поставленной задачи.

Основной особенностью OpenGL является простота приложения. Ядро OpenGL контролирует процесс обработки примитивов. Для передачи данных используется процедурная модель. В каждый момент времени состояние OpenGL определяется через набор переменных, которые задают параметры обработки. Каждый новый элемент проходит обработку в соответствии с текущим состоянием. Такой механизм весьма эффективен, а код обычно короток и прост. При использовании ядра OpenGL совместно с объектноориентированными технологиями сложностей не возникает.

Структура DirectX значительно отличается от OpenGL. DirectX прежде всего основано на модели COM (Component Object Model). Это означает, что в отличие от обычного вызова функций, эта модель предполагает выполнение дополнительных действий, связанных с компонентной архитектурой DirectX. B частности, код, в котором используются вызовы DirectX, обычно не является легко читаемым и понимаемым.

Серьезным достоинством OpenGL является то, что нет ограничения к доступу ядра. Другими словами, программист, может использовать все функции библиотеки, а также дописывать новые. В отношении к DirectX, то здесь 
ситуация прямо противоположная. Только Microsoft может вносить какие-либо изменения в библиотеку.

\section{Постановка требований}

Исходя из выше сказанного для наилучшей генерации ландшафта предлагается использовать графическую библиотеку OpenGL.

При ландшафтной генерации необходимо выполнить ряд требований:

1. Выводимый ландшафт должен быть довольно больших размеров, и строиться из карты высот размеров в $4 \mathrm{~K} \cdot 4 \mathrm{~K}$, где К - это количество точек, выводимых на экран.

2. Результаты должны хранится не в памяти персонального устройства, а динамически подгружаться по необходимости. Результат должен сводится к минимуму предзагрузок и ожиданий.

3. Полученный ландшафт должен быть эффективным, другими словами данные должны отсылаться на графический процессор (ГПУ) большими блоками, и обновляться как можно реже при необходимости.

4. Сетка высот должна учитывать кривизну ландшафта, меняя детализацию по необходимости.

\section{Решение поставленных требований}

Все перечисленные выше требования решаются по схеме, состоящей из мелких картинок, имеющих одинаковый размер, но соответствующих территории разных размеров. Аналогична схема для ландшафта. На ней происходит разбиение ландшафта на квадратные области, каждая область представляется регулярной сеткой, имеет почти одинаковое число треугольников, и рисуется за один дип, но при этом соответствует территории разных размеров (рисунок 1 c)). 

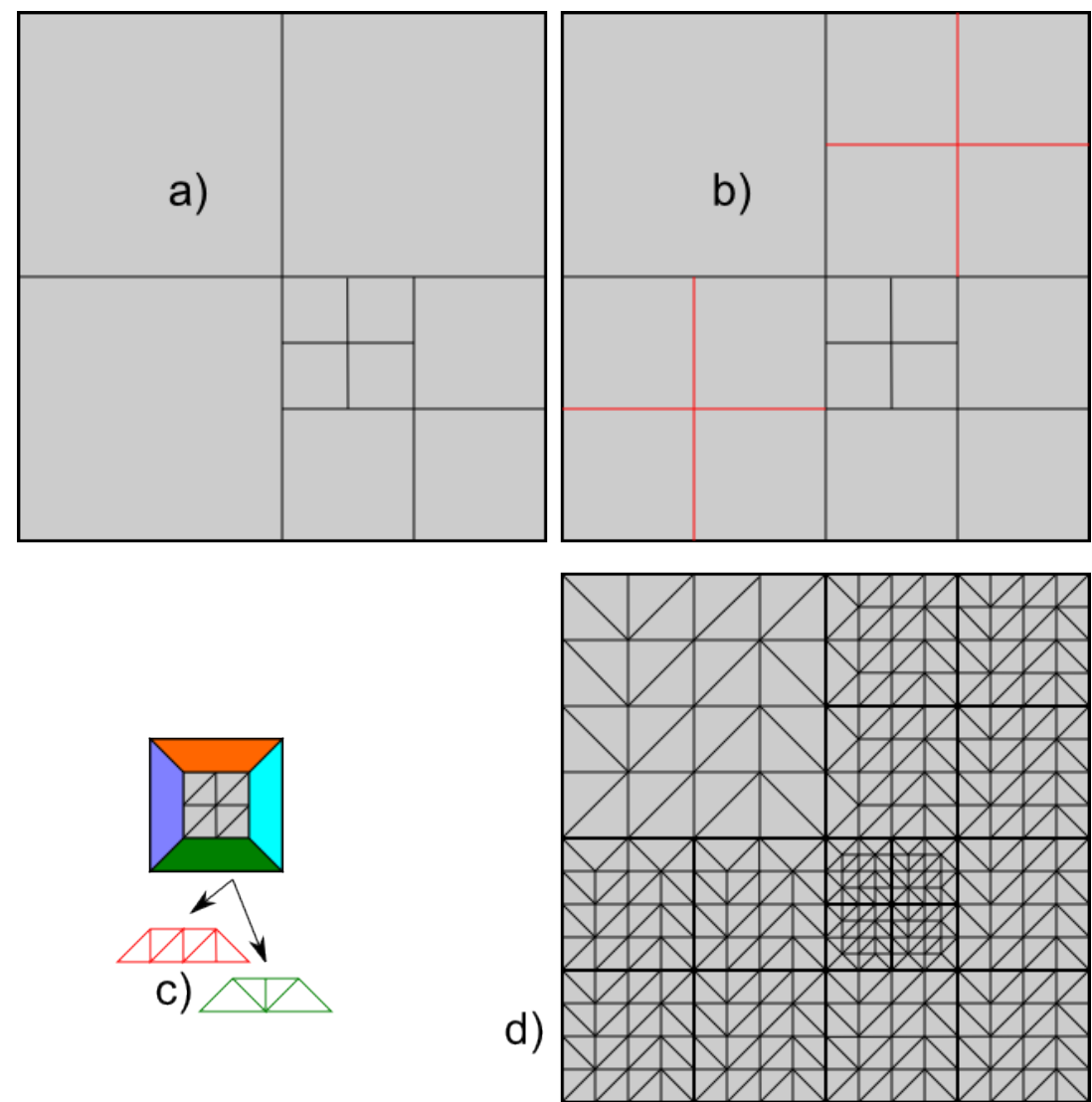

Рисунок 1 - Схема генеращчи ландшафта

Ландшафт рисуется в несколько дипов, в каждом из которых примерно одинаковое число треугольников (рисунок 1, d). Структура сетки представляет собой квадродерево. Представим себе для начала большой квадрат, размер которого в метрах равен размеру нашего ландшафта. Этот квадрат будет корнем квадродерева. Функцией, отвечающей за увеличение карты ландшафта, является morph-фактор. Если значение этого фактора больше единицы, то есть необходимость разбить узел дерева на четыре дочерних узла. Значения функции могут зависеть как от положения камеры, так и от кривизны поверхности ландшафта.

Для нахождения значения увеличения карты ландшафта morph-фактора используется формула:

$$
\text { factor }=\frac{\text { Height }}{2 \cdot \operatorname{tg}(\text { fov } / 2)} \text {; }
$$

где factor - данные высот содержащие приблизительный размер узла в пикселях на экране;

Height - высота до максимальной точки объекта ландшафта; fov - набор данных содержащих координаты расположения текстуры. 
Далее определим, какой будет уровень детализации, при приближении карты пользователем:

$$
\begin{aligned}
& \operatorname{morph}_{x}=\text { Min }\left(1, \frac{\text { size }_{x}}{\text { MaxTriSize } \cdot \text { TERNODESIZE }}-0,5\right) \cdot 2 ; \\
& \operatorname{morphT}_{y}=\text { Min }\left(1, \frac{\text { size }_{y}}{\text { MaxTriSize } \cdot \text { TERNODESIZE }}-0,5\right) \cdot 2 \text {; }
\end{aligned}
$$

где morphTx, morphTy - определяет уровень детализации, заданный пользователем;

sizex, sizey, sizez - приблизительный размер сторон узла в пикселях на экране; MaxTriSize максимальный размер треугольников в пикселях на экране; TERNODESIZE - расстояние расположение координат [X, Y]. В которой Xкомпонента каждого элемента буфера является шириной в какой-то точке для узла, Y-компонента является высота, полученная из lod с меньшей детализацией.

Уровень сглаживания объектов ландшафта расположенных по осям после его увеличения, определяется по следующим формулам:

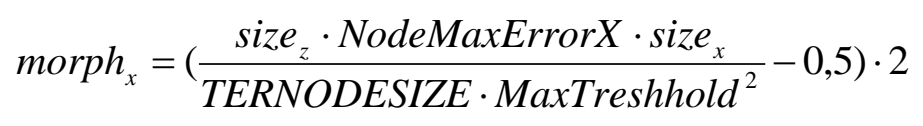

NodeMaxErrorX - максимальное количество ошибок по оси X;

MaxTreshhold - определяет максимальную детализацию ландшафта;

morphx, morphy - «сглаживание» объекта ландшафта после его увеличения.

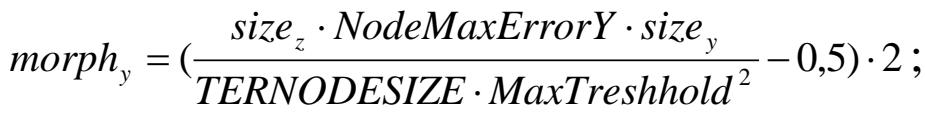

$$
\begin{aligned}
& \operatorname{morph}=\operatorname{Max}\left(\operatorname{Max}\left(\operatorname{morphT}_{x}, \operatorname{morph}_{x}\right), \operatorname{Max}\left(\operatorname{morph} T_{y}, \operatorname{morph}_{y}\right)\right) ;
\end{aligned}
$$

где NodeMaxErrorУ - максимальное количество ошибок по оси У.

Разбиения продолжаются до тех пор, пока morph-фактор не станет меньше единицы. Каждый листовой квадрат дерева разбивается на треугольники и образует один дип.

На границе квадратов разных размеров для упрощения их стыковки друг с другом без просветов вводится дополнительное правило. Любой квадрат может одной стороной граничить не более, чем с двумя другими квадратами, и при возникновении таких ситуаций производится дополнительные разбиения квадратов на треугольники.

Вычислим размер сторон узла в пикселях на экране, для того, чтобы изображение соответствовало разрешению, установленному пользователем: 


$$
\text { size }_{x}=\text { factor } \cdot \text { Scale }_{x} \cdot \frac{|\vec{R} \cdot \vec{i}|}{R^{2}} \text {; }
$$

где $\vec{i}, \vec{j}, \vec{k}$ - элементы массива данных;

$\mathrm{R}-$ радиус.

$$
\begin{aligned}
& \text { size }_{y}=\text { factor } \cdot \text { Scale }_{y} \cdot \frac{|\vec{R} \cdot \vec{j}|}{R^{2}} ; \\
& \text { size }_{z}=\text { factor } \cdot \text { Scale }_{z} \cdot \frac{|\vec{R} \cdot \vec{k}|}{R^{2}} ;
\end{aligned}
$$

Разбиение квадратов на треугольники для отрисовки представлется в виде центральной части и краёв, как показано на рисунке 1 с). Краем может быть сетка разряженная (показана зелённым), так и неразряжённая (показана красным цветом). Всего 4 края, по два варианта в каждом, и тем самым общее число вариантов сеток для квадрата равно 24=16. При выборе сетки для края квадрата используется правило: если сторона дипа граничит с квадратами такой же детализации, или более, то выбираем детализированную сетку (красную), если сторона дипа граничит с прямоугольниками меньшей детализации, то выбираем разрежённую сетку (зелённую). Итоговая сетка показана на рисунке $1 \mathrm{~d}$ ).

В итоге имеем 16 вариантов сеток для квадратов - 16 разных заранее приготовленных (и, например, оптимизированных с помощью функции NVTriStrip) индексных буферов (на самом деле один, из 16 частей, к которым обращаемся по смещению). Каждому листовому прямоугольнику из бинарного дерево соответствует карта высот, размером $(\mathrm{N}+1) \cdot(\mathrm{N}+1)$, где $\mathrm{N}-$ константа, равная на рисунке 4 , и в каждом дипе примерно $\mathrm{N} \cdot \mathrm{N} \cdot 2$ треугольника. Если $\mathrm{N}=$ 32 , и число треугольников в дипе 2 тыс. При рендеринге вначале заполняется буфер вершины высотами для каждого прямоугольника. Размер буфера будет равен $(\mathrm{N}+1) \cdot(\mathrm{N}+1) \cdot($ число листовых прямоугольников), т.е. довольно небольшим. Затем для каждого прямоугольника по дипу, указываем необходимое смещение в вершинном буфере. При перестройке дерева возможно, что часть нужных высот уже будет находиться в буфере, поэтому полной перезаливки данных каждый элемент сохраненного буфера элемента не требует, нужно только заменить ненужные блоки из $(\mathrm{N}+1) \cdot(\mathrm{N}+1)$ вершин на новые.

Предположим, что имеется ландшафт размером в 4K·4K точек. Тогда и карта нормалей будет таких же размеров. И при увеличении размера она может не поместиться целиком в виде текстуры. Поэтому используем библиотеку big_texture, которая разбивает текстуры на блоки одинаковых размеров 
(например, 256.256 пикселей). Они имеют также, как и блоки высот, координаты: lod - уровень детализации текстур; х - ширина узла, у - высота узла, и для каждого элемента при прорисовке выбирается соответствующий блок текстуры.

Формула удаленности пользователя от предмета по соответствующим осям представлена ниже:

$$
\text { Scale }_{x}=\frac{\text { TerrainSizeX }}{2^{\text {lod }}} \text {; }
$$

где Scalex - удаленность пользователя от предмета по оси х; TerrainSizeX - размерность ландшафта по оси х.

$$
\text { Scale }_{y}=\frac{\text { TerrainSizeY }}{2^{\text {lod }}}
$$

$$
\vec{R}=\overrightarrow{\text { SomeNodePoint }}-\overrightarrow{\text { CamPos }} \text {. }
$$

Где Scaley - удаленность пользователя от предмета по оси у;

$\vec{R}$ - размерность;

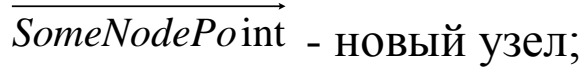

$\overrightarrow{\mathrm{CamPos}}$ - позиция камеры;

TerrainSizeY - размерность ландшафта по оси у.

\section{Заключение}

В данной статье рассмотрены актуальные графические библиотеки DirectX и OpenGL для реализации объемной ландшафтной генерации. На основе сравнительного анализа наиболее приемлемой библиотекой для выполнения постарения ландшафта предложено использовать графическую библиотеку OpenGL. Приведены все этапы создания ландшафта, начиная от разработки идеи и концепции, и, заканчивая схемой построения ландшафтной карты. Изучен инструментарий, при помощи которого генерируется ландшафт, что позволяет использовать эту технологию в тренажерно-обучающих системах для отработки навыков в условиях реального времени. 
1. William L. Raffe, Fabio Zambetta, and Xiaodong Li «A Survey of Procedural Terrain Generation Techniques using Evolutionary Algorithms», 2012.

2. Адамс Д. DirectX: продвинутая анимация. Комплект. - «КУДИЦ-ПРЕСС», 2004. — С. 480. — ISBN 59579-0025-7.

3. Шардаков В.M. Обработка динамических потоков мультимедийных данных в 3D моделировании/ В.М. Шардаков// Наука и бизнес: Пути развития. - 2016. - № 12. с. 4245.

4. Эйнджел Э. Интерактивная компьютерная графика. Вводный курс на базе OpenGL, 2 изд. Пер. с англ.- Москва, «Вильямс», 2001.

5. Порев В.Н Компьютерная графика. - СПб., ВНV, 2002, 432 с.

6. Шикин А. В., Боресков А. В. Компьютерная графика. Полигональные модели. М.: ДИАЛОГ-МИФИ, 2001, 464 с.

7. Тихомиров Ю. Программирование трехмерной графики. СПб, ВНV, 1998, 246 с.

8. Performance OpenGL: Platform Independent Techniques. SIGGRAPH 2001 course [Электронный https://www.opengl.org/archives/resources/code/samples/s2001/perfogl.pdf.

9. OpenGL performance optimization, Siggraph’97 course. [Электронный ресурс] https://www.cs.montana.edu/courses/fall2004/425/course24.pdf

10. Баяковский Ю.M., Игнатенко А.В. Начальный курс OpenGL. М.: Планета знаний, 2007, $221 \mathrm{c}$.

11. Segal M., Akeley K. The OpenGL. Graphics System. USA: Silicon Graphics, Ins, 2004, 382 p. 